\title{
Gedanken und Überzeugungen werden überschätzt
}

\author{
Ein Interview mit Adrian Wells
}

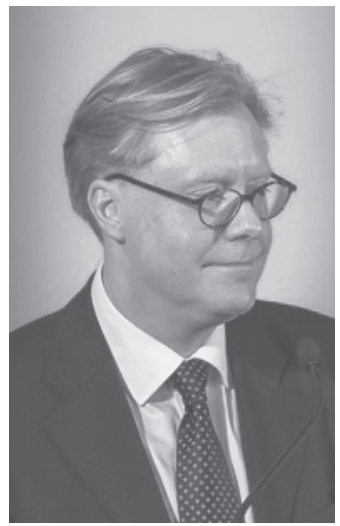

Adrian Wells ist klinischer Psychologe und Professor für klinische und experimentelle Psychopathologie an der Universität Manchester. Er lernte Kognitive Therapie bei Aaron T. Beck in Philadelphia. In den 1990er Jahren entwickelte er die Metakognitive Therapie (MCT). Grundannahme der MCT ist, dass psychische Probleme durch bestimmte Denk-, Aufmerksamkeits- und Verhaltensmuster, das sogenannte Kognitive Aufmerksamkeitssyndrom (CAS), und darauf bezogene Metakognitionen (also Gedanken über Gedanken) aufrechterhalten werden [Wells, 2009]. Die MCT wird häufig zur «dritten Welle der Verhaltenstherapie» gezählt. In Großbritannien und in Norwegen gibt es mit der "MCT Masterclass" seit wenigen Jahren ein 2-jähriges Lehrangebot. 2014 wird die Masterclass nun auch in Deutschland angeboten. Aber was genau ist MCT? Ist sie nicht einfach nur eine besondere Form der Kognitiven Verhaltenstherapie (CBT)? Oder gar alter Wein in neuen Schläuchen? - Dr. Michael Simons fragte nach.

Was ist Metakognitive Therapie (MCT) - und insbesondere: Was ist MCT im Vergleich zur CBT?

MCT ist ein evidenzbasierter Behandlungsansatz, der auf einem spezifischen Informationsverarbeitungsmodell psychischer Störungen basiert. Ich werde oft gefragt, ob MCT eine Form der CBT ist, aber ich denke nicht, dass dies eine sehr hilfreiche Frage ist. Beide Ansätze haben im weiteren Sinne mit Kognitionen zu tun, sehen aber unterschiedliche kognitive Aspekte als entscheidend und haben unterschiedliche Behandlungstechniken entwickelt, die nicht immer miteinander kompatibel sind. Der Unterschied kann verdeutlicht werden, indem man sich eines der zentralen Prinzipien der CBT ansieht: Was eine Person denkt, ist wichtig und kann zu Störungen führen. Die Aussage der MCT ist jedoch anders: nicht die Gedanken sind von Bedeutung, sondern die Reaktionen der Person darauf. Daher überprüft der MCT-Therapeut nicht den Wahrheitsgehalt der Gedanken, sondern schaut stattdessen, wie die Person ihr Denken als Antwort auf negative Gedanken und Überzeugungen reguliert. Es ist wichtig, sich klarzumachen, dass negative Gedanken und Überzeugungen häufig vorkommen und normale Erfahrungen sind, die in der
Regel vorübergehen. Das metakognitive Modell postuliert, dass diese Gedanken das Erleben dominieren und zu Störungen führen durch die Art und Weise, wie eine Person darauf antwortet. Es ist egal, ob ein Gedanke valide oder verzerrt, oder ob er negativ oder positiv ist. Entscheidend ist, ob die Person wählen kann, so mit den Gedanken umzugehen, dass eine mentale Fixierung verhindert und das Denken als eine Form der Selbstregulation nicht überbewertet wird.

Kritiker könnten sagen, dass Ihre letzte Aussage die Entwicklung der CBT unterschätzt, und auch die Entwicklung der MCT aus der CBT. In Ihren Büchern [z.B. Wells, 1997, 2000] nannten Sie Ihren Ansatz noch stets "Kognitive Therapie». Wäre es nicht möglich, MCT als ein Mitglied der "CBT-Familie»zu sehen, genauso wie Dialektisch Behaviorale Therapie (DBT), Schematherapie, Akzeptanz- und Commitment-Therapie $(A C T)$, usw.?

Um diese Frage richtig beantworten zu können, ist es hilfreich, etwas zu den Hintergründen und der Theorie der MCT zu sagen. Das Modell besagt, dass ein Denkstil, der dominiert wird von Sich-Sorgen-Machen, Grübeln und Bedrohungs-

\section{KARGER}

Fax +497614520714

Information@Karger.com

www.karger.com

\section{(c) 2014 S. Karger GmbH, Freiburg}

Accessible online at:

www.karger.com/ver 
monitoring als Reaktion auf negative Gedanken und Überzeugungen, ungünstige emotionale Reaktionen verstärkt und verlängert. Dieser Denkstil wird von Metakognitionen angetrieben, in denen positive Überzeugungen (z.B. «Mich sorgen bedeutet, dass ich vorbereitet bin.» und «Meine Gefühle $\mathrm{zu}$ analysieren wird mir helfen, meine Depression loszuwerden.») und negative Überzeugungen («Ich habe die Kontrolle über mein Denken verloren.» und «Gedanken könnten mir schaden.») entscheidend sind. Insofern sich die MCT mit Kognitionen beschäftigt, ist sie auf eine Art Kognitive Therapie. Aber wesentliche Unterschiede bestehen darin, wie die MCT mit den Kognitionen umgeht und welche Veränderungstechniken sie nutzt. Diese Unterschiede zu verstehen, ist wichtiger als sie zu verschleiern, indem man beide in eine Schublade steckt. Dies soll auf keinen Fall den Beitrag der CBT abwerten, die immer eine wichtige Option ist und bleiben wird. Dennoch bleibt es wichtig, neuere und potenziell effektivere Behandlungen zu entwickeln, und ich glaube, dass das Unvermögen, die Unterschiede zwischen den Modellen und Techniken zu erkennen, den Prozess in diesem Feld verzögern wird. Vielleicht kann ich eine Metapher benutzen, um zu veranschaulichen, was ich meine: Niemand würde abstreiten, dass ein Fahrrad und ein Boot das gleiche sind, oder? Schließlich sind sie beide ein Transportmittel; aber natürlich verschleiert diese Ebene der Analyse sehr wichtige Unterschiede.

In meinem Buch von 1997 «Cognitive Therapy of Anxiety Disorders» [Wells, 1997] habe ich die metakognitive Behandlung für die generalisierte Angststörung (GAS) und für Zwänge neben die kognitiven Behandlungen für andere Angststörungen gestellt. Das lag daran, dass die kognitive Behandlung dieser beiden Störungen weit hinter den Ergebnissen lag, die wir für CBT bei z.B. Panik oder sozialer Angst erwarten konnten, und aufgrund der sich abzeichnenden Resultate war etwas anderes nötig. Es wäre genauer gewesen, das Buch «Cognitive and Metacognitive Therapy» zu nennen, aber es schien wahrscheinlich, dass dies Probleme machen würde, da der Begriff «Metakognitive Therapie» in dieser Zeit nicht gebräuchlich war. Ich hatte bereits über mehrere Jahre erheblichen Widerstand erfahren bei dem Versuch, metakognitive Konstrukte zu veröffentlichen, und ich wollte versuchen, einige Barrieren zu entfernen und ein Behandlungsmanual anbieten, das es Klinikern und Wissenschaftlern ermöglichen würde, mit diesem neuen Ansatz zu experimentieren. Ich denke, diese Strategie hat funktioniert; z.B. wird MCT für GAS jetzt in den «NICE Clinical Guidelines» zitiert, weil Wissenschaftler mit der Evaluierung weitergekommen sind. Trotzdem muss ich zugeben, dass es seine Nachteile gehabt haben könnte, MCT in ein explizites CBT-Buch aufzunehmen, weil das die wichtigen Unterschiede vernachlässigt. Also: MCT ist eine Variante der CBT, aber da der Begriff CBT heutzutage so unspezifisch ist, müssen wir präziser verdeutlichen, welche Version wir meinen. MCT ist sicherlich nicht dasselbe wie CBT, was jedoch nicht bedeutet, dass wir
Letzteres abwerten müssten. Aus empirischer Sicht sind Vergleiche zwischen MCT und CBT (einige werden momentan gemacht) wichtig, um die Unterschiede zu überprüfen.

In den letzten Jahren sehen wir die Entwicklung von «DritteWelle»-Therapien. In einem neuen Cochrane-Review [Hunot et al., 2013$]$ über Dritte-Welle-Therapien für Depression wird auch MCT darunter subsummiert; interessanterweise mit der Bemerkung: «Obwohl MCT normalerweise als ein 〈Dritte Welle $\rangle-$ CBT-Ansatz angesehen wird, berichtet Hofmann (2008), dass Adrian Wells dies nicht so sieht.»Können Sie uns verdeutlichen, wo Sie die Unterschiede und Gemeinsamkeiten zwischen MCT und der dritten Welle sehen?

Der Begriff «dritte Welle» wurde benutzt, um Ansätze zusammenzufassen wie «Mindfulness Based Cognitive Therapy» (MBCT), ACT und DBT. Diese Ansätze haben eine Anzahl von Gemeinsamkeiten, hauptsächlich im Gebrauch von Strategien, die auf buddhistischen Meditationspraktiken basieren. MCT benutzt diese Strategien nicht und hat eine Reihe von therapeutischen Techniken entwickelt, die von einer spezifischen Theorie abgeleitet wurden. MBCT hat zentrale Elemente der CBT, wie Gedanken hinterfragen und beibehalten, und benutzt Meditationstechniken als Rückfallprävention. Dies unterscheidet sich von der MCT, in der Gedanken nicht hinterfragt werden, und die andere Techniken zur Rückfallprävention nutzt. ACT fokussiert auf Werte in der Therapie, was kein Fokus in der MCT ist. ACT hat keinen Plan zur Symptomreduktion, wohingegen ein vorrangiges Anliegen der MCT ist, Symptome zu mildern und Genesung zu bewirken. Das gemeinsame Merkmal dieser Ansätze und der MCT ist, dass alle darauf fokussieren, Patienten zu befähigen, einen neue Art der Beziehung zu ihren Gedanken aufzubauen; aber man kann argumentieren, dass CBT dies auch tut. Einer der wesentlichen Unterschiede zwischen MCT und diesen anderen Ansätzen ist, dass nur MCT metakognitive Überzeugungen beinhaltet und gezielt versucht, diese zu modifizieren. MCT ist der einzige Ansatz in dieser Gruppe, der auf einem hoch spezifizierten Apriori-Modell der Informationsverarbeitung basiert.

Einige Wissenschaftler wie z.B. Wampold [z.B. 2001] verkünden das "Dodo Bird Verdict». Sie sagen, dass es keinen Unterschied macht, welcher Therapierichtung man folgt, und dass es wichtiger ist, wie man Therapie macht, mit einer Betonung auf allgemeine Wirkfaktoren. Was denken Sie darüber? Macht es etwas aus, welchem Therapieprotokoll man folgt?

Ja, ich glaube es macht einen Unterschied, welchem Therapieansatz man folgt. Wenn eine Behandlung darauf abzielt, gut spezifizierte kausale Faktoren zu korrigieren, dann ist sie logischerweise besser. Es gibt deutliche Hinweise darauf, dass einige Behandlungsansätze, insbesondere die für Angststörungen, wirksamer sind. Zum Beispiel zeigen die Ergeb- 
nisse gut durchgeführter Studien, dass CBT effektiver ist als angewandte Entspannung für Patienten mit Panikstörung, dass MCT effektiver ist als CBT bei Patienten mit GAS, und wir haben einige vorläufige Ergebnisse, die zeigen, dass MCT bei posttraumatischer Belastungsstörung (PTBS) effektiver oder schneller sein könnte als prolongierte Exposition. Zusätzlich zeigen Studien über spezifische Behandlungstechniken, die als Komponentenstudien (dismantling studies) angesehen werden können, dass eine Expositionsbehandlung nach dem Habituationsrational weniger effektiv ist als Exposition in Kombination mit der Veränderung von Sicherheitsverhalten oder mit der Modifizierung der Aufmerksamkeitsfokussierung des Patienten [Wells et al., 1995; Wells und Papageorgiou, 1998]. Diese Strategien sind Komponenten des effektivsten Therapieansatzes für soziale Phobie. Die Daten stützen überzeugend die Idee, dass das, was wir tun, den Unterschied in der Behandlung ausmacht. Vielleicht können wir das unspezifische Argument umdrehen: Die Qualität der therapeutischen Beziehung ist wichtig, aber hilfreich für eine starke therapeutische Beziehung ist, die effektivsten Behandlungstechniken zu nutzen. Das heißt nicht, dass die Beziehung und die Art und Weise, wie Therapie durchgeführt wird, irrelevant sind. Nach meiner persönlichen Erfahrung sind die Verfügbarkeit von Behandlungsmanualen, Supervision und das Ausmaß der Therapeutenkompetenz Faktoren, die einen Unterschied für die Therapieeffekte machen können.

Sie haben die Behandlung von Angststörungen erwähnt. Es gibt zunehmende Hinweise zur Expositionstherapie, dass Habituation für den Behandlungserfolg gar nicht so wichtig ist. Neuere Erkenntnisse [z.B. Craske et al., 2008] und einige neurobiologisch orientierte Kliniker und Forscher betonen, dass Veränderung Wiederholung benötigt; man lernt neues Verhalten, indem man es in verschiedenen Kontexten wiederholt. Kann es sein, dass die MCT die Wichtigkeit von Verhaltensveränderungen und von Wiederholungen unterschätzt?

In der MCT ist die Verhaltensänderung eine zentrale Komponente der Behandlung. Die Verhaltensweisen, die modifiziert werden, sind insbesondere die, die zu Konflikten in der Selbstregulation führen und/oder maladaptive Metakognitionen aufrechterhalten. Habituation oder die Veränderung von Überzeugungen auf dem kognitiven Level werden dabei nicht als Mechanismen der Veränderung betrachtet. Vielmehr gehen wir davon aus, dass Verhaltensveränderung effektiv ist, wenn sie das CAS auflöst, metakognitive Überzeugungen verändert und die flexible Kontrolle verbessert. Zum Beispiel, in einem Fall mit Zwangsstörung, sieht die MCT eine kurze Exposition vor (5 min); die geschieht dann mehrere Male (3-5 Mal) und dient als Test für Metakognitionen oder als eine Gelegenheit, Kontrolle über das Sich-Sorgen-Machen zu üben [Fisher und Wells, 2005]. Dieses Modell bedeutet, dass es grundverschiedene Wege der Verhaltensveränderung gibt.
Beispielsweise ist es in der Behandlung von Zwangsstörungen nicht unbedingt notwendig, die Durchführung von Ritualen zu verhindern.

Dem Ansatz der MCT zufolge sollte das Ziel sein, dem Patienten dabei zu helfen herauszufinden, dass seine Gedanken nicht wichtig sind und er die andauernde Beschäftigung mit diesen Gedanken aufgeben kann. Dem Patienten kann erlaubt werden zu kontrollieren, um mit dem expliziten Ziel herauszufinden, dass Zweifel oder negative Gedanken bedeutungslos sind und es daher sicher ist, sie in Ruhe zu lassen. Tatsächlich verändert der MCT-Therapeut das Selbstregulationsziel des Patienten davon weg, Ängste durch Kontrollieren zu reduzieren (Sorgen zu beenden), und stattdessen hin zum Kontrollieren, um etwas sehr Nützliches in Bezug auf Gedanken zu lernen. Vielleicht führt Ritualprävention im Kontext von Exposition auch dazu, die Bedeutung von Gedanken neu zu bewerten, tut dies aber eher zufällig und weniger effizient. Exposition und Reaktionsverhinderung können genutzt werden, aber dann dazu, Überzeugungen über Gedanken zu testen, sodass die Expositionsdauer sehr kurz ist. Gestützt wird dies durch Befunde, die belegen, dass traditionelle Expositionsbehandlungen Veränderung auf metakognitiver Ebene bewirken, und dass metakognitive Exposition sehr kurz sein kann [Solem et al., 2009].

Sie fragen auch nach Wiederholung. Die ist auch relevant bei der Durchführung von MCT. Wiederholung wird nicht mit dem Ziel der Habituation verwendet, sondern wird als sinnvoll erachtet, um neue Strategien einzuüben, auf Gedanken zu antworten. Ein Grundgedanke der MCT ist, dass Wissen prozedural oder als «Wissen wie» gespeichert wird, und dies wird durch das «Tun» gelernt und verstärkt. Diese Art des Wissens, insbesondere das Wissen in Bezug auf die Kontrolle von Kognitionen und Aufmerksamkeit, sollte direkt erlebt und eingeübt werden. Wiederholung verbessert metakognitives Bewusstsein, ermöglicht es den Klienten, typische Auslöser des CAS zu identifizieren, das CAS in seinen verschiedenen Formen zu erkennen und die flexible exekutive Kontrolle und die Entscheidung über die Antwort zu stärken.

MCT nimmt an, dass sich eine Depression entwickelt, wenn jemand exzessiv grübelt und glaubt, dass er nicht damit aufhören kann. Aber manchmal sagen depressive Patienten, sie grübelten gar nicht. Was würden Sie einem solchen Patienten sagen oder was würden Sie ihn fragen?

Grübeln kann ohne unmittelbares Bewusstsein stattfinden. Das Bewusstsein für diesen Prozess kann auf verschiedene Art und Weise verändert sein. Wenn Grübeln chronisch im Hintergrund auftritt, muss die Person lernen, diesen Prozess zu erkennen. In vielen Fällen wird dieser Prozess als die Lösung für das Problem der schlechten Stimmung angesehen, und der Betroffene glaubt, so die Gründe der Traurigkeit und der negativen Erfahrungen zu finden. Unter diesen Umständen wird Grübeln nicht als eine problematische Antwort be- 
trachtet, sondern als Versuch, die Situation zu meistern. Laut des metakognitiven Modells fokussiert die Aufmerksamkeit eines depressiven Patienten auf Bedrohungen, und Grübeln wird von ihm nicht als eine Gefahrenquelle erkannt, so wie das Sich-Sorgen-Machen als eine Bedrohung für eine Person mit beispielsweise GAS erlebt wird. So kann das Bewusstsein für das Grübeln und das Problemverständnis verringert werden. Wenn es schwierig ist, das Grübeln zu erkennen, können bestimmte Fragen nützlich sein, so wie: «Wie viel Zeit in der letzten Woche haben Sie damit zugebracht, darüber nachzudenken, wie Sie sich fühlen und warum Sie sich so fühlen?» «Haben Sie bei sich bemerkt, dass Sie über die Vergangenheit nachdenken oder darüber, wie Sie sich fühlen?» «Wie oft haben Sie sich selber gefragt: Warum fühle ich mich so? Was wäre wenn...? Warum ich? Wird es je aufhören?» Wenn der metakognitive Therapeut solche Fragen stellt, ist es nützlich, die Häufigkeit und Dauer dieser Denkepisoden zu erfragen und somit metakognitives Bewusstsein zu steigern.

\section{Was sind mögliche Schwächen und Grenzen der MCT?}

Ich denke, es ist noch zu früh, die Schwächen zu erkennen, dafür brauchen wir noch mehr Forschung und besonders unabhängige, gut kontrollierte Studien für verschiedene Erkran- kungen. Es hat sehr lange gedauert, diese Behandlungsmethode zu entwickeln, aber ich glaube, dass wir an der Schwelle zu einer Behandlung sind, die die CBT übertreffen kann, zumindest bei einigen Erkrankungen. Aber es gibt eine Schwäche: MCT erfordert eine konzeptuelle Anpassung der CBTDenkweise, damit Therapeuten die Modelle und Techniken nicht durcheinanderbringen und kombinieren. Beides zu kombinieren birgt das Risiko, dass die Vorteile der MCT verloren gehen könnten.

Abschließende Frage: Wie sehen Sie die Zukunft der MCT? Was kommt als Nächstes?

Einer der nächsten Schritte in der Entwicklung der MCT wird die Analyse von Daten großer randomisierter und kontrollierter Studien aus den Bereichen Depression, GAS und Leiden herz- oder krebskranker Patienten sein. Die Anwendung der Therapie auf Kinder und Jugendliche und auf psychotische Patienten wird sich weiterentwickeln. Von besonderem Interesse sind weitere Arbeiten zur Neuropsychologie mit MCT-Techniken und die Entwicklung von Frühinterventionen bzw. Prävention.

Professor Wells, ich danke Ihnen sehr für dieses Gespräch!

\section{Literatur}

Craske MG, Kircanski K, Zelikowsky M, Mystkowski J, Chowdhury N, Baker A: Optimizing inhibitory learning during exposure therapy. Behav Res Ther 2008;46:5-27.

Fisher PL, Wells A: Experimental modification of beliefs in obsessive-compulsive disorder: a test of the metacognitive model. Behav Res Ther 2005;43:821-829.

-Hunot V, Moore TH, Caldwell DM, Furukawa TA, Davies P, Jones H, Honyashiki M, Chen P, Lewis G, Churchill R: 'Third wave' cognitive and behavioural therapies versus other psychological therapies for depression. Cochrane Database Syst Rev 2013;18:10.
Solem S, Håland AT, Vogel PA, Hansen B, Wells A: Change in metacognitions predicts outcome in obsessive-compulsive disorder patients undergoing treatment with exposure and response prevention. Behav Res Ther 2009;47:301-307.

Wampold BE: The Great Psychotherapy Debate. Models, Methods, and Findings. New York, Taylor and Francis, 2001.

Wells A: Cognitive Therapy of Anxiety Disorders. A Practice and Conceptual Guide. Chichester, Wiley, 1997.
Wells A: Emotional Disorders and Metacognition: Innovative Cognitive Therapy. Chichester, Wiley, 2000.

Wells A: Metacognitive Therapy for Anxiety and Depression. New York, Guilford Press, 2009.

Wells A, Clark DM, Salkovskis PM, Ludgate J, Hackmann A, Gelder M: Social phobia: the role of insituation safety behaviors in maintaining anxiety and negative beliefs. Behav Ther 1995:26;153-161.

Wells A, Papageorgiou C: Social phobia: effects of external attention on anxiety, negative beliefs and perspective taking. Behav Ther 1998;29:357-370. 